\title{
An optimal three-point eighth-order iterative method without memory for solving nonlinear equations with its dynamics
}

\author{
Gunar Matthies $^{a *} \quad$ Mehdi Salimi ${ }^{b, c \dagger} \quad$ Somayeh Sharifi ${ }^{d \ddagger}$ \\ Juan Luis Varona ${ }^{e \S}$ \\ ${ }^{a}$ Institut für Numerische Mathematik, Technische Universität Dresden, Germany \\ ${ }^{b}$ Center for Dynamics, Department of Mathematics, Technische Universität Dresden, Germany \\ ${ }^{c}$ Department of Mathematics, Universiti Putra Malaysia, 43400 UPM Serdang, Selangor, Malaysia \\ ${ }^{d}$ MEDAlics, Research Center at Università per Stranieri Dante Alighieri, Reggio Calabria, Italy \\ ${ }^{e}$ Departamento de Matemáticas y Computación, Universidad de La Rioja, Logroño, Spain
}

\begin{abstract}
We present a three-point iterative method without memory for solving nonlinear equations in one variable. The proposed method provides convergence order eight with four function evaluations per iteration. Hence, it possesses a very high computational efficiency and supports Kung and Traub's conjecture. The construction, the convergence analysis, and the numerical implementation of the method will be presented. Using several test problems, the proposed method will be compared with existing methods of convergence order eight concerning accuracy and basin of attraction. Furthermore, some measures are used to judge methods with respect to their performance in finding the basin of attraction.
\end{abstract}

Keywords: Optimal multi-point iterative methods; Simple root; Order of convergence; Kung and Traub's conjecture; Basins of attraction.

Mathematics Subject Classification: 65H05, 37F10

\section{Introduction}

Solving nonlinear equations is a basic and extremely valuable tool in all fields in science and engineering. One can distinguish between two general approaches for solving nonlinear equations numerically, namely, one-point and multi-point methods. The basic optimality theorem shows that an analytic one-point method based on $k$ evaluations is of order at most $k$, see [27, §5.4] or [15] for an improved proof. The Newton-Raphson method $x_{n+1}:=x_{n}-\frac{f\left(x_{n}\right)}{f^{\prime}\left(x_{n}\right)}$ is probably the most widely used algorithms for finding roots. It requires two evaluations per iteration step, one for $f$ and one for $f^{\prime}$, and results in second order convergence which is optimal for this one-point method.

Some computational issues encountered by one-point methods are overcome by multi-point methods since they allow to achieve greater accuracy with the same number of function evaluations. Important aspects related to these methods are convergence order and efficiency. It is favorable to attain with a fixed number of function evaluations per iteration step a convergence order which is as high as possible. A central role in this context plays the unproved conjecture by Kung and Traub [15] which states that an optimal multi-point method without memory provides a convergence order of $2^{k}$ while

\footnotetext{
*gunar.matthies@tu-dresden.de

${ }^{\dagger}$ mehdi.salimi@tu-dresden.de

${ }^{\ddagger}$ somayeh.sharifi@medalics.org

§jvarona@unirioja.es
} 
using $k+1$ evaluations in each iteration step. The efficiency index for a method with $k$ evaluations and convergence order $p$ and $k$ evaluations is given by $E(k, p)=\sqrt[k]{p}$, see [19]. Hence, the efficiency of a method supporting Kung and Traub's conjecture is $\sqrt[k+1]{2^{k}}$. In particular, an optimal method with convergence order eight has an efficiency index $\sqrt[4]{8} \simeq 1.68179$.

A large number of multi-point methods for finding simple roots of a nonlinear equation $f(x)=0$ with a scalar function $f: D \subset \mathbb{R} \rightarrow \mathbb{R}$ which is defined on an open interval $D$ (or $f: D \subset \mathbb{C} \rightarrow \mathbb{C}$ defined on a region $D$ in the complex plane $\mathbb{C}$ ) have been developed and analyzed for improving the convergence order of classical methods like the Newton-Raphson iteration.

Some well known two-point methods without memory are described e.g. in Jarratt [13], King [14], and Ostrowski [19]. Using inverse interpolation, Kung and Traub [15] constructed two general optimal classes without memory. Since then, there have been many attempts to construct optimal multi-point methods, utilizing e.g. weight functions, see in particular [2,3,5, 16, 20 24, 26, 29].

We will construct a three-point method of convergence order eight which is free from second order derivatives, uses 4 evaluations, and provides the efficiency index $\sqrt[4]{8} \simeq 1.68179$.

A wide used criterion to judge and rank different methods for solving nonlinear equations is the basin of attraction. We will use two measures to assess the performance in finding the basin of attraction 28.

The paper is organized as follows. Section 2 introduces the new methods based on a Newton step and Newton's interpolation. Moreover, details of the new method and the proof of its optimal convergence order eight are given. The numerical performance of the proposed method compared to other methods are illustrated in Section 3. We approximate and visualize the basins of attraction in Section 4 for the proposed method and several existing methods, both graphically and by mean of introduced numerical performance measures 28 . Finally, we conclude in Section 5 .

\section{Description of the method and convergence analysis}

We construct in this section a new optimal three-point method for solving nonlinear equations by using a Newton-step and Newton's interpolation polynomial of degree three which was also applied in $[22]$.

Method 1: The new method is given by

$$
\left\{\begin{aligned}
y_{n} & :=x_{n}-u_{n}\left(x_{n}\right), \\
z_{n} & :=x_{n}-u_{n}\left(x_{n}\right)\left(1+\frac{f\left(y_{n}\right)}{f\left(x_{n}\right)}+\left(1+\frac{1}{1+u_{n}\left(x_{n}\right)}\right)\left(\frac{f\left(y_{n}\right)}{f\left(x_{n}\right)}\right)^{2}\right), \\
x_{n+1} & :=z_{n}-\frac{f\left(z_{n}\right)}{f\left[z_{n}, y_{n}\right]+\left(z_{n}-y_{n}\right) f\left[z_{n}, y_{n}, x_{n}\right]+\left(z_{n}-y_{n}\right)\left(z_{n}-x_{n}\right) f\left[z_{n}, y_{n}, x_{n}, x_{n}\right]},
\end{aligned}\right.
$$

where $u_{n}\left(x_{n}\right)=\frac{f\left(x_{n}\right)}{f^{\prime}\left(x_{n}\right)}$. The standard notation for divided differences in Newton's interpolation

$$
g\left[t_{\nu}, t_{\nu+1}, \ldots, t_{\nu+j}\right]=\frac{g\left[t_{\nu+1}, \ldots, t_{\nu+j}\right]-g\left[t_{\nu}, \ldots, t_{\nu+j-1}\right]}{t_{\nu+j}-t_{\nu}},
$$

with $g\left[t_{\nu}\right]=g\left(t_{\nu}\right)$ and $g\left[t_{\nu}, t_{\nu}\right]=g^{\prime}\left(t_{\nu}\right)$ are used.

The iteration method (2.1) and all forthcoming methods are applied for $n=0,1, \ldots$ where $x_{0}$ denotes an initial approximation of the simple root $x^{*}$ of the function $f$. The method (2.1) uses four evaluations per iteration step, three for $f$ and one for $f^{\prime}$. Note that 2.1 works for real and complex functions.

The convergence order of method $(2.1)$ is given in the following theorem. 
Theorem 1. Let $f: D \subset \mathbb{R} \rightarrow \mathbb{R}$ be an eight times continuously differentiable function with a simple zero $x^{*} \in D$. If the initial point $x_{0}$ is sufficiently close to $x^{*}$ then the method defined by (2.1) converges to $x^{*}$ with order eight.

Proof. Let $e_{n}:=x_{n}-x^{*}, e_{n, y}:=y_{n}-x^{*}, e_{n, z}:=z_{n}-x^{*}$ and $c_{n}:=\frac{f^{(n)}\left(x^{*}\right)}{n ! f^{\prime}\left(x^{*}\right)}$ for $n \in \mathbb{N}$. Using the fact that $f\left(x^{*}\right)=0$, the Taylor expansion of $f$ at $x^{*}$ yields

$$
f\left(x_{n}\right)=f^{\prime}\left(x^{*}\right)\left(e_{n}+c_{2} e_{n}^{2}+c_{3} e_{n}^{3}+\cdots+c_{8} e_{n}^{8}\right)+O\left(e_{n}^{9}\right)
$$

and

$$
f^{\prime}\left(x_{n}\right)=f^{\prime}\left(x^{*}\right)\left(1+2 c_{2} e_{n}+3 c_{3} e_{n}^{2}+4 c_{4} e_{n}^{3}+\cdots+9 c_{9} e_{n}^{8}\right)+O\left(e_{n}^{9}\right) .
$$

Therefore, we have

$$
\begin{aligned}
\frac{f\left(x_{n}\right)}{f^{\prime}\left(x_{n}\right)}=e_{n} & -c_{2} e_{n}^{2}+\left(2 c_{2}^{2}-2 c_{3}\right) e_{n}^{3}+\left(-4 c_{2}^{3}+7 c_{2} c_{3}-3 c_{4}\right) e_{n}^{4} \\
& +\left(8 c_{2}^{4}-20 c_{2}^{2} c_{3}+6 c_{3}^{2}+10 c_{2} c_{4}-4 c_{5}\right) e_{n}^{5} \\
& +\left(-16 c_{2}^{5}+52 c_{2}^{3} c_{3}-28 c_{2}^{2} c_{4}+17 c_{3} c_{4}-c_{2}\left(33 c_{3}^{2}-13 c_{5}\right)\right) e_{n}^{6}+O\left(e_{n}^{7}\right),
\end{aligned}
$$

and

$$
\begin{aligned}
e_{n, y}=y_{n}-x^{*}= & c_{2} e_{n}^{2}+\left(-2 c_{2}^{2}+2 c_{3}\right) e_{n}^{3}+\left(4 c_{2}^{3}-7 c_{2} c_{3}+3 c_{4}\right) e_{n}^{4} \\
& +\left(-8 c_{2}^{4}+20 c_{2}^{2} c_{3}-6 c_{3}^{2}-10 c_{2} c_{4}+4 c_{5}\right) e_{n}^{5} \\
& +\left(16 c_{2}^{5}-52 c_{2}^{3} c_{3}+28 c_{2}^{2} c_{4}-17 c_{3} c_{4}+c_{2}\left(33 c_{3}^{2}-13 c_{5}\right)\right) e_{n}^{6}+O\left(e_{n}^{7}\right) .
\end{aligned}
$$

We have

$$
f\left(y_{n}\right)=f^{\prime}\left(x^{*}\right)\left(e_{n, y}+c_{2} e_{n, y}^{2}+c_{3} e_{n, y}^{3}+\cdots+c_{8} e_{n, y}^{8}\right)+O\left(e_{n, y}^{9}\right)
$$

by a Taylor expansion of $f$ at $x^{*}$. By substituting (2.2)-(2.4) into (2.1), we get

$$
\begin{aligned}
e_{n, z}=z_{n}-x^{*}=c_{2} & \left(c_{2}+5 c_{2}^{2}-c_{3}\right) e_{n}^{4} \\
& +\left(-8 c_{2}^{3}-36 c_{2}^{4}-2 c_{3}^{2}+c_{2}^{2}\left(-1+32 c_{3}\right)+c_{2}\left(4 c_{3}-2 c_{4}\right)\right) e_{n}^{5}+O\left(e_{n}^{6}\right) .
\end{aligned}
$$

We obtain

$$
f\left(z_{n}\right)=f^{\prime}\left(x^{*}\right)\left(e_{n, z}+c_{2} e_{n, z}^{2}+c_{3} e_{n, z}^{3}+\cdots+c_{8} e_{n, z}^{8}\right)+O\left(e_{n, z}^{9}\right)
$$

by using again a Taylor expansion of $f$ at $x^{*}$. Substituting (2.2)-(2.5) into (2.1), we get

$$
e_{n+1}=x_{n+1}-x^{*}=c_{2}^{2}\left(c_{2}+5 c_{2}^{2}-c_{3}\right)\left(c_{2}^{2}-5 c_{2}^{3}-c_{2} c_{3}+c_{4}\right) e_{n}^{8}+O\left(e_{n}^{9}\right),
$$

which finishes the proof of the theorem.

We will compare the new method (2.1) with some existing optimal three-point methods of order eight having the same optimal computational efficiency index equal to $\sqrt[4]{8} \simeq 1.68179$, see $[19,27$.

The existing methods that we are going to use to compare are the following:

Method 2: The method by Chun and Lee [5] is given by

$$
\left\{\begin{aligned}
y_{n} & :=x_{n}-\frac{f\left(x_{n}\right)}{f^{\prime}\left(x_{n}\right)}, \\
z_{n} & :=y_{n}-\frac{f\left(y_{n}\right)}{f^{\prime}\left(x_{n}\right)} \cdot \frac{1}{\left(1-\frac{f\left(y_{n}\right)}{f\left(x_{n}\right)}\right)^{2}}, \\
x_{n+1} & :=z_{n}-\frac{f\left(z_{n}\right)}{f^{\prime}\left(x_{n}\right)} \cdot \frac{1}{\left(1-H\left(t_{n}\right)-J\left(s_{n}\right)-P\left(u_{n}\right)\right)^{2}}
\end{aligned}\right.
$$


with weight functions

$$
H\left(t_{n}\right)=-\beta-\gamma+t_{n}+\frac{t_{n}^{2}}{2}-\frac{t_{n}^{3}}{2}, \quad J\left(s_{n}\right)=\beta+\frac{s_{n}}{2}, \quad P\left(u_{n}\right)=\gamma+\frac{u_{n}}{2},
$$

where $t_{n}=\frac{f\left(y_{n}\right)}{f\left(x_{n}\right)}, s_{n}=\frac{f\left(z_{n}\right)}{f\left(x_{n}\right)}, u_{n}=\frac{f\left(z_{n}\right)}{f\left(y_{n}\right)}$, and $\beta, \gamma \in \mathbb{R}$. Note that the parameters $\beta$ and $\gamma$ cancel when used in (2.7). Hence, their choice has no contribution to the method.

Method 3: The method by B. Neta [17, see also [18, formula (9)], is given by

$$
\left\{\begin{aligned}
y_{n} & :=x_{n}-\frac{f\left(x_{n}\right)}{f^{\prime}\left(x_{n}\right)} \\
z_{n} & :=y_{n}-\frac{f\left(x_{n}\right)+A f\left(y_{n}\right)}{f\left(x_{n}\right)+(A-2) f\left(y_{n}\right)} \cdot \frac{f\left(y_{n}\right)}{f^{\prime}\left(x_{n}\right)}, \quad A \in \mathbb{R} \\
x_{n+1} & :=y_{n}+\delta_{1} f^{2}\left(x_{n}\right)+\delta_{2} f^{3}\left(x_{n}\right),
\end{aligned}\right.
$$

where

$$
\begin{aligned}
F_{y} & =f\left(y_{n}\right)-f\left(x_{n}\right), & F_{z} & =f\left(z_{n}\right)-f\left(x_{n}\right), \\
\zeta_{y} & =\frac{1}{F_{y}}\left(\frac{y_{n}-x_{n}}{F_{y}}-\frac{1}{f^{\prime}\left(x_{n}\right)}\right), & \zeta_{z} & =\frac{1}{F_{z}}\left(\frac{z_{n}-x_{n}}{F_{z}}-\frac{1}{f^{\prime}\left(x_{n}\right)}\right), \\
\delta_{2} & =-\frac{\zeta_{y}-\zeta_{z}}{F_{y}-F_{z}}, & \delta_{1} & =\zeta_{y}+\delta_{2} F_{y} .
\end{aligned}
$$

We will use $A=0$ in the numerical experiments of this paper.

Method 4: The Sharma and Sharma method [24] is given by

$$
\left\{\begin{aligned}
y_{n} & :=x_{n}-\frac{f\left(x_{n}\right)}{f^{\prime}\left(x_{n}\right)}, \\
z_{n} & :=y_{n}-\frac{f\left(y_{n}\right)}{f^{\prime}\left(x_{n}\right)} \cdot \frac{f\left(x_{n}\right)}{f\left(x_{n}\right)-2 f\left(y_{n}\right)}, \\
x_{n+1} & :=z_{n}-\frac{f\left[x_{n}, y_{n}\right] f\left(z_{n}\right)}{f\left[x_{n}, z_{n}\right] f\left[y_{n}, z_{n}\right]} W\left(t_{n}\right),
\end{aligned}\right.
$$

with the weight function

$$
W\left(t_{n}\right)=1+\frac{t_{n}}{1+\alpha t_{n}}, \quad \alpha \in \mathbb{R},
$$

and $t_{n}=\frac{f\left(z_{n}\right)}{f\left(x_{n}\right)}$. We will use $\alpha=1$ in the numerical experiments of this paper.

Method 5: The method from Babajee, Cordero, Soleymani and Torregrosa 2] is given by

$$
\left\{\begin{aligned}
y_{n} & :=x_{n}-\frac{f\left(x_{n}\right)}{f^{\prime}\left(x_{n}\right)}\left(1+\left(\frac{f\left(x_{n}\right)}{f^{\prime}\left(x_{n}\right)}\right)^{5}\right), \\
z_{n} & :=y_{n}-\frac{f\left(y_{n}\right)}{f^{\prime}\left(x_{n}\right)}\left(1-\frac{f\left(y_{n}\right)}{f\left(x_{n}\right)}\right)^{-2}, \\
x_{n+1} & :=z_{n}-\frac{f\left(z_{n}\right)}{f^{\prime}\left(x_{n}\right)} \cdot \frac{1+\left(\frac{f\left(y_{n}\right)}{f\left(x_{n}\right)}\right)^{2}+5\left(\frac{f\left(y_{n}\right)}{f\left(x_{n}\right)}\right)^{4}+\frac{f\left(z_{n}\right)}{f\left(y_{n}\right)}}{\left(1-\frac{f\left(y_{n}\right)}{f\left(x_{n}\right)}-\frac{f\left(z_{n}\right)}{f\left(x_{n}\right)}\right)^{2}} .
\end{aligned}\right.
$$


Method 6: The method from Thukral and Petković [26] is given by

$$
\left\{\begin{aligned}
y_{n} & :=x_{n}-\frac{f\left(x_{n}\right)}{f^{\prime}\left(x_{n}\right)}, \\
z_{n} & :=y_{n}-\frac{f\left(y_{n}\right)}{f^{\prime}\left(x_{n}\right)} \cdot \frac{f\left(x_{n}\right)+\beta f\left(y_{n}\right)}{f\left(x_{n}\right)+(\beta-2) f\left(y_{n}\right)}, \quad \beta \in \mathbb{R}, \\
x_{n+1} & :=z_{n}-\frac{f\left(z_{n}\right)}{f^{\prime}\left(x_{n}\right)} \cdot\left(\varphi\left(t_{n}\right)+\psi\left(s_{n}\right)+\omega\left(u_{n}\right)\right),
\end{aligned}\right.
$$

where weight functions are

$$
\varphi\left(t_{n}\right)=\left(1+\frac{t_{n}}{1-2 t_{n}}\right)^{2}, \quad \psi\left(s_{n}\right)=\frac{s_{n}}{1-\alpha s_{n}}, \quad \alpha \in \mathbb{R}, \quad \omega\left(u_{n}\right)=4 u_{n},
$$

and $t_{n}=\frac{f\left(y_{n}\right)}{f\left(x_{n}\right)}, s_{n}=\frac{f\left(z_{n}\right)}{f\left(y_{n}\right)}$ and $u_{n}=\frac{f\left(z_{n}\right)}{f\left(x_{n}\right)}$. We will use $\beta=0$ and $\alpha=1$ in the numerical experiments of this paper.

\section{Numerical examples}

The new three-point method (2.1) is tested on several nonlinear equations. To obtain high accuracy and avoid the loss of significant digits, we employed multi-precision arithmetic with 20000 significant decimal digits in the programming package Mathematica.

We are going to perform numerical experiments with the four test functions $f_{j}, j=1, \ldots, 4$, which appear in Table 1 . We are going to reach the given root $x^{*}$ starting with the mentioned $x_{0}$ for the four functions and the six methods of convergence order eight.

\begin{tabular}{lcc}
\hline test function $f_{j}$ & root $x^{*}$ & initial guess $x_{0}$ \\
\hline$f_{1}(x)=\ln \left(1+x^{2}\right)+e^{x^{2}-3 x} \sin x$ & 0 & 0.35 \\
$f_{2}(x)=1+e^{2+x-x^{2}}+x^{3}-\cos (1+x)$ & -1 & -0.3 \\
$f_{3}(x)=\left(1+x^{2}\right) \cos \frac{\pi x}{2}+\frac{\ln \left(x^{2}+2 x+2\right)}{1+x^{2}}$ & -1 & -1.1 \\
$f_{4}(x)=x^{4}+\sin \frac{\pi}{x^{2}}-5$ & $\sqrt{2}$ & 1.5 \\
\hline
\end{tabular}

Table 1: Test functions $f_{1}, \ldots, f_{4}$, root $x^{*}$, and initial guess $x_{0}$.

In order to test our proposed method (2.1) and compare it with the methods (2.7)-(2.11), we compute the error, the computational order of convergence (COC) by the approximate formula 30

$$
\mathrm{COC} \approx \frac{\ln \left|\left(x_{n+1}-x^{*}\right) /\left(x_{n}-x^{*}\right)\right|}{\ln \left|\left(x_{n}-x^{*}\right) /\left(x_{n-1}-x^{*}\right)\right|}
$$

and the approximated computational order of convergence (ACOC) by the formula 6

$$
\mathrm{ACOC} \approx \frac{\ln \left|\left(x_{n+1}-x_{n}\right) /\left(x_{n}-x_{n-1}\right)\right|}{\ln \left|\left(x_{n}-x_{n-1}\right) /\left(x_{n-1}-x_{n-2}\right)\right|} .
$$

It is worth noting that COC has been used in the recent years. Nevertheless, ACOC is more practical because it does not require to know the root $x^{*}$. See [10] for a comparison among several convergence orders. Note that these formulas may result for particular examples in convergence 
orders which are higher than expected. The reason is that the error equation (2.6) contains problemdependent coefficients which may vanish for some nonlinear functions $f$. However, the formulas (3.1) and (3.2) will provide for a "random" example good approximations for the convergence order of the method.

We have used both COC and ACOC to check the accuracy of the considered methods. Note that both $\mathrm{COC}$ and $\mathrm{ACOC}$ give already for small values of $n$ good experimental approximations to convergence order.

The comparison of our method (2.1) with the methods (2.7)-(2.11) applied to the four nonlinear equations $f_{j}(x)=0, j=1, \ldots, 4$, are presented in in Table 2. We abbreviate (2.1) by M1 and 2.7 - 2.11) as M2-M6, respectively. The computational convergence order COC and ACOC are given $n=3$. Note that they are for all problems and methods in excellent with the theoretical order of convergence.

\begin{tabular}{cllllll}
\hline \multicolumn{1}{c}{ M1 } & \multicolumn{1}{c}{ M2 } & \multicolumn{1}{c}{ M3 } & \multicolumn{1}{c}{ M4 } & \multicolumn{1}{c}{ M5 } & \multicolumn{1}{c}{ M6 } \\
\hline$f_{1}, x_{0}=0.35$ & & & & & & \\
$\left|x_{1}-x^{*}\right|$ & $0.610 \mathrm{e}-6$ & $0.721 \mathrm{e}-4$ & $0.893 \mathrm{e}-4$ & $0.753 \mathrm{e}-4$ & $0.347 \mathrm{e}-3$ & $0.328 \mathrm{e}-3$ \\
$\left|x_{2}-x^{*}\right|$ & $0.319 \mathrm{e}-46$ & $0.230 \mathrm{e}-30$ & $0.126 \mathrm{e}-30$ & $0.619 \mathrm{e}-31$ & $0.471 \mathrm{e}-25$ & $0.256 \mathrm{e}-25$ \\
$\left|x_{3}-x^{*}\right|$ & $0.179 \mathrm{e}-368$ & $0.252 \mathrm{e}-242$ & $0.200 \mathrm{e}-245$ & $0.128 \mathrm{e}-247$ & $0.546 \mathrm{e}-200$ & $0.345 \mathrm{e}-202$ \\
COC & 8.0000 & 8.0000 & 8.0000 & 8.0000 & 8.0000 & 8.0000 \\
ACOC & 8.0000 & 7.9999 & 7.9999 & 7.9999 & 7.9999 & 7.9999 \\
\hline$f_{2}, x_{0}=-0.3$ & & & & & \\
$\left|x_{1}-x^{*}\right|$ & $0.248 \mathrm{e}-3$ & $0.157 \mathrm{e}-3$ & $0.763 \mathrm{e}-4$ & $0.871 \mathrm{e}-4$ & $0.411 \mathrm{e}-3$ & $0.273 \mathrm{e}-4$ \\
$\left|x_{2}-x^{*}\right|$ & $0.582 \mathrm{e}-32$ & $0.119 \mathrm{e}-33$ & $0.540 \mathrm{e}-35$ & $0.134 \mathrm{e}-34$ & $0.377 \mathrm{e}-29$ & $0.321 \mathrm{e}-38$ \\
$\left|x_{3}-x^{*}\right|$ & $0.532 \mathrm{e}-261$ & $0.138 \mathrm{e}-274$ & $0.342 \mathrm{e}-284$ & $0.438 \mathrm{e}-281$ & $0.189 \mathrm{e}-237$ & $0.117 \mathrm{e}-309$ \\
COC & 8.0000 & 8.0000 & 8.0000 & 8.0000 & 8.0000 & 8.0000 \\
ACOC & 8.0000 & 7.9998 & 7.9999 & 7.9999 & 7.9999 & 7.9999 \\
\hline$f_{3}, x_{0}=-1.1$ & & & & & \\
$\left|x_{1}-x^{*}\right|$ & $0.106 \mathrm{e}-7$ & $0.614 \mathrm{e}-8$ & $0.388 \mathrm{e}-8$ & $0.175 \mathrm{e}-8$ & $0.554 \mathrm{e}-8$ & $0.100 \mathrm{e}-7$ \\
$\left|x_{2}-x^{*}\right|$ & $0.482 \mathrm{e}-63$ & $0.328 \mathrm{e}-65$ & $0.254 \mathrm{e}-67$ & $0.154 \mathrm{e}-70$ & $0.426 \mathrm{e}-66$ & $0.136 \mathrm{e}-63$ \\
$\left|x_{3}-x^{*}\right|$ & $0.833 \mathrm{e}-506$ & $0.217 \mathrm{e}-523$ & $0.877 \mathrm{e}-541$ & $0.582 \mathrm{e}-567$ & $0.528 \mathrm{e}-531$ & $0.154 \mathrm{e}-510$ \\
COC & 8.0000 & 8.0000 & 8.0000 & 8.0000 & 8.0000 & 8.0000 \\
ACOC & 7.9999 & 8.0000 & 7.9999 & 8.0000 & 8.0000 & 7.9999 \\
\hline$f_{4}, x_{0}=1.5$ & & & & & \\
$\left|x_{1}-x^{*}\right|$ & $0.148 \mathrm{e}-7$ & $0.433 \mathrm{e}-8$ & $0.327 \mathrm{e}-10$ & $0.642 \mathrm{e}-10$ & $0.281 \mathrm{e}-8$ & $0.727 \mathrm{e}-10$ \\
$\left|x_{2}-x^{*}\right|$ & $0.138 \mathrm{e}-61$ & $0.134 \mathrm{e}-66$ & $0.369 \mathrm{e}-84$ & $0.101 \mathrm{e}-81$ & $0.341 \mathrm{e}-68$ & $0.543 \mathrm{e}-81$ \\
$\left|x_{3}-x^{*}\right|$ & $0.769 \mathrm{e}-494$ & $0.116 \mathrm{e}-534$ & $0.967 \mathrm{e}-676$ & $0.389 \mathrm{e}-656$ & $0.161 \mathrm{e}-547$ & $0.530 \mathrm{e}-650$ \\
COC & 8.0000 & 8.0000 & 8.0000 & 8.0000 & 8.0000 & 8.0000 \\
ACOC & 8.0000 & 7.9999 & 7.9999 & 7.9999 & 8.0000 & 7.9999 \\
\hline
\end{tabular}

Table 2: Errors, COC, and ACOC for the iterative methods (2.1) and (2.7)-(2.11) (abbreviated as M1-M6) applied to the find the root of test functions $f_{1}, \ldots, f_{4}$ given in Table 1 .

\section{Dynamic behavior}

We have already observed that all methods converge if the initial guess is chosen suitably. We now investigate the regions where the initial point has to chosen in order to achieve the root. In other words, we will numerically approximate the domain of attraction of the zeros as a qualitative measure of how the method depends on the choice of the initial approximation of the root. To answer this 
important question on the dynamical behavior of the algorithms, we will investigate the dynamics of the new method (2.1) and compare it with the methods (2.7)-(2.11).

Let's recall some basic concepts such as basin of attraction. For more details and many other examples of the study of the dynamic behavior of iterative methods, one can consult $1,2,4,7,9,11$, $12,25,28$.

Let $Q: \mathbb{C} \rightarrow \mathbb{C}$ be a rational map on the complex plane. For $z \in \mathbb{C}$, we define its orbit as the set $\operatorname{orb}(z)=\left\{z, Q(z), Q^{2}(z), \ldots\right\}$. The convergence $\operatorname{orb}(z) \rightarrow z^{*}$ is understood in the sense $\lim _{k \rightarrow \infty} Q^{k}(z)=z^{*}$. A point $z_{0} \in \mathbb{C}$ is called periodic point with minimal period $m$ if $Q^{m}\left(z_{0}\right)=z_{0}$ where $m$ is the smallest positive integer with this property (and thus $\left\{z_{0}, Q\left(z_{0}\right), \ldots, Q^{m-1}\left(z_{0}\right)\right\}$ is a cycle). A periodic point with minimal period 1 is called fixed point. Moreover, a periodic point $z_{0}$ with period $m$ is called attracting if $\left|\left(Q^{m}\right)^{\prime}\left(z_{0}\right)\right|<1$, repelling if $\left|\left(Q^{m}\right)^{\prime}\left(z_{0}\right)\right|>1$, and neutral otherwise. The Julia set of a nonlinear map $Q(z)$, denoted by $J(Q)$, is the closure of the set of its repelling periodic points. The complement of $J(Q)$ is the Fatou set $F(Q)$.

The six methods (2.1) and (2.7)-(2.11) provide iterative rational maps $Q$ when they are applied to find roots of complex polynomials $p$. In particular, we are interested in the basins of attraction of the roots of the polynomials where the basin of attraction of a root $z^{*}$ is the complex set $\left\{z_{0} \in \mathbb{C}\right.$ : $\left.\operatorname{orb}\left(z_{0}\right) \rightarrow z^{*}\right\}$. It is well known that the basins of attraction of the different roots lie in the Fatou set $F(Q)$. The Julia set $J(Q)$ is, in general, a fractal and the rational map $Q$ is unstable there.

For the dynamical and graphical point of view, we take a $600 \times 600$ grid of the square $[-3,3] \times$ $[-3,3] \subset \mathbb{C}$ and assign a color to each point $z_{0} \in D$ according to the simple root to which the corresponding orbit of the iterative method starting from $z_{0}$ converges, and we mark the point as black if the orbit does not converge to a root in the sense that after at most 15 iterations it has a distance to any of the roots which is larger than $10^{-3}$. We have used only 15 iterations because we are using methods of convergence order eight which, if they converge, do this very fast. The basins of attraction are distinguished by their color.

\begin{tabular}{|c|c|}
\hline Test polynomials & Roots \\
\hline$p_{1}(z)=z^{2}-1$ & $1, \quad-1$ \\
\hline$p_{2}(z)=z^{3}-z$ & $0, \quad 1, \quad-1$ \\
\hline$p_{3}(z)=z\left(z^{2}+1\right)\left(z^{2}+4\right)$ & $0, \quad 2 i, \quad-2 i, \quad i, \quad-i$ \\
\hline$p_{4}(z)=\left(z^{4}-1\right)\left(z^{2}+2 i\right)$ & $1, \quad i, \quad-1, \quad-i, \quad-1+i, \quad 1-i$ \\
\hline$p_{5}(z)=z^{7}-1$ & $k=0, \ldots, 6$ \\
\hline$p_{6}(z)=\left(10 z^{5}-1\right)\left(z^{5}+10\right)$ & $\left(\frac{1}{10}\right)^{1 / 5} e^{2 k \pi i / 5}, \quad(-10)^{1 / 5} e^{2 k \pi i / 5}, \quad k=0, \ldots, 4$ \\
\hline
\end{tabular}

Table 3: Test polynomials $p_{1}(z), \ldots, p_{6}(z)$ and their roots.

Different colors are used for different roots. In the basins of attraction, the number of iterations needed to achieve the root is shown by the brightness. Brighter color means less iteration steps. Note that black color denotes lack of convergence to any of the roots. This happens, in particular, when the method converges to a fixed point that is not a root or if it ends in a periodic cycle or at infinity. Actually and although we have not done it in this paper, infinity can be considered an ordinary point if we consider the Riemann sphere instead of the complex plane. In this case, we can assign a new "ordinary color" for the basin of attraction of infinity. Details for this idea can be found in [12].

Basins of attraction for the six methods $(2.1)$ and $(2.7)-(2.11)$ for the six test problems $p_{i}(z)=0$, $i=1, \ldots, 6$, are illustrated in Figures 16 from left to right and from top to bottom.

From the pictures, we can easily judge the behavior and suitability of any method depending on the circumstances. If we choose an initial point $z_{0}$ in a zone where different basins of attraction touch each other, it is impossible to predict which root is going to be reached by the iterative method that 

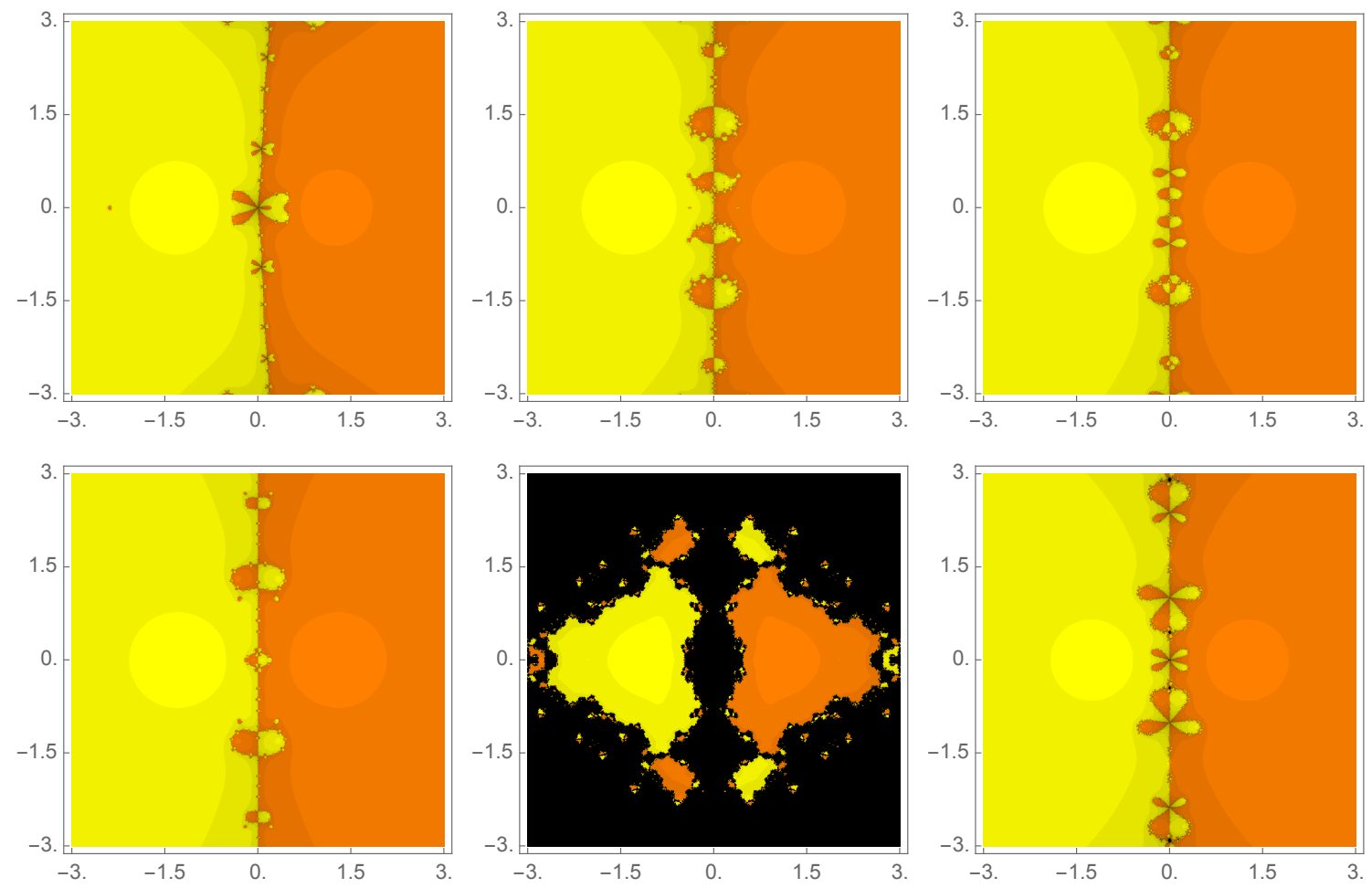

Figure 1: Comparison of basins of attraction of methods (2.1) and (2.7)- 2.11) for the test problem $p_{1}(z)=z^{2}-1=0$.
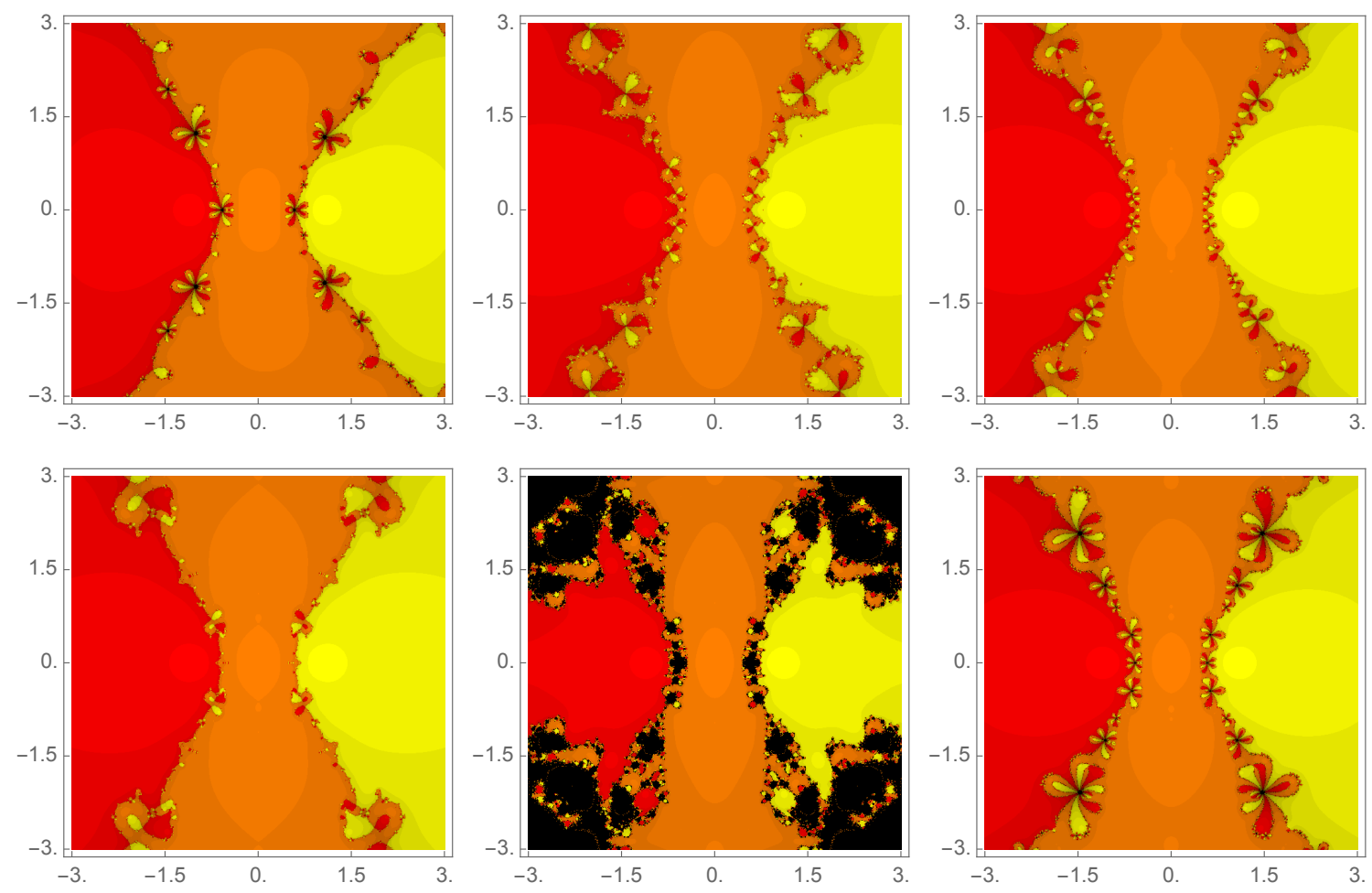

Figure 2: Comparison of basins of attraction of methods (2.1) and (2.7)-(2.11) for the test problem $p_{2}(z)=z^{3}-z=0$. 

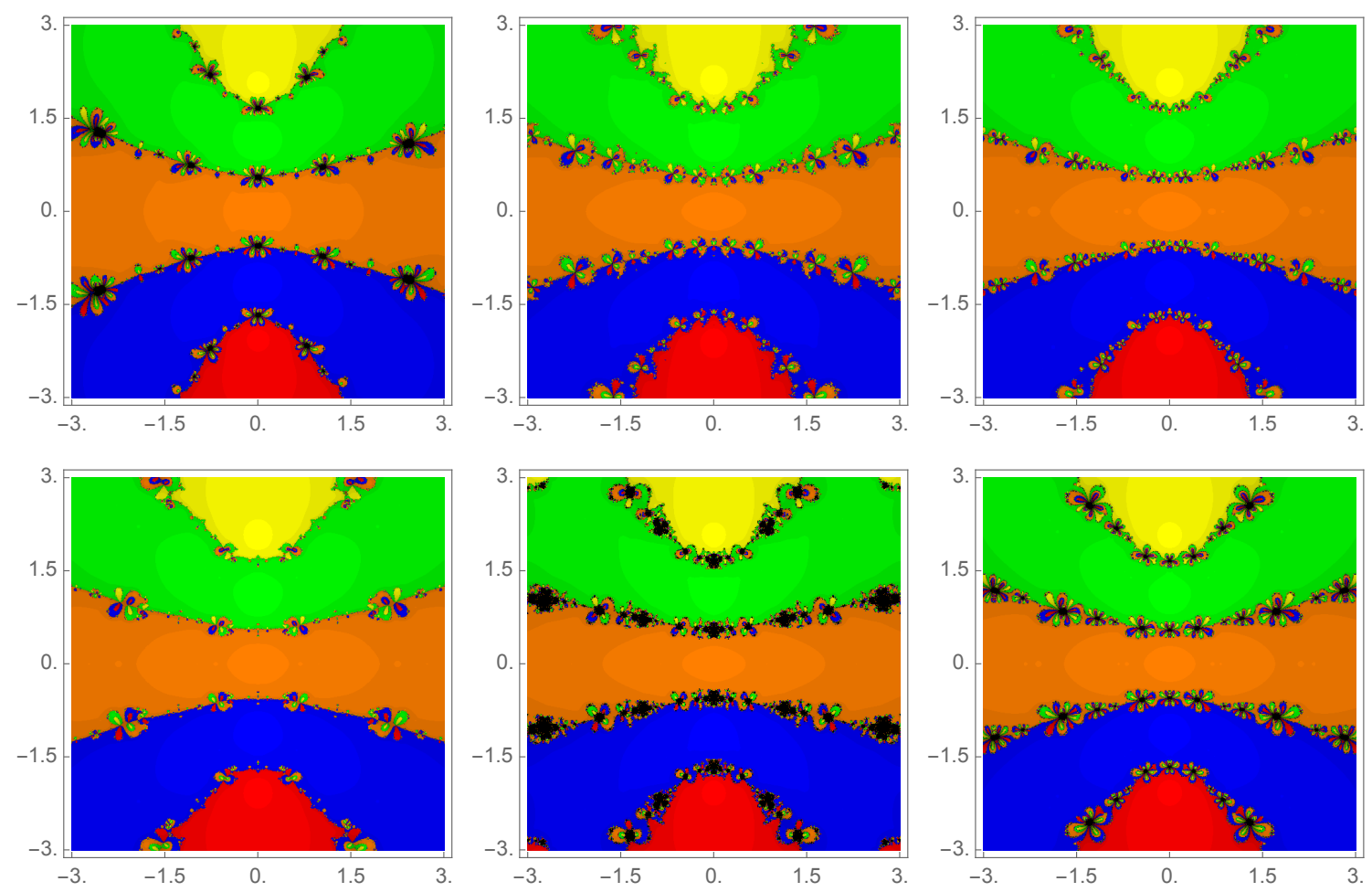

Figure 3: Comparison of basins of attraction of methods (2.1) and (2.7)-(2.11) for the test problem $p_{3}(z)=z\left(z^{2}+1\right)\left(z^{2}+4\right)=0$.
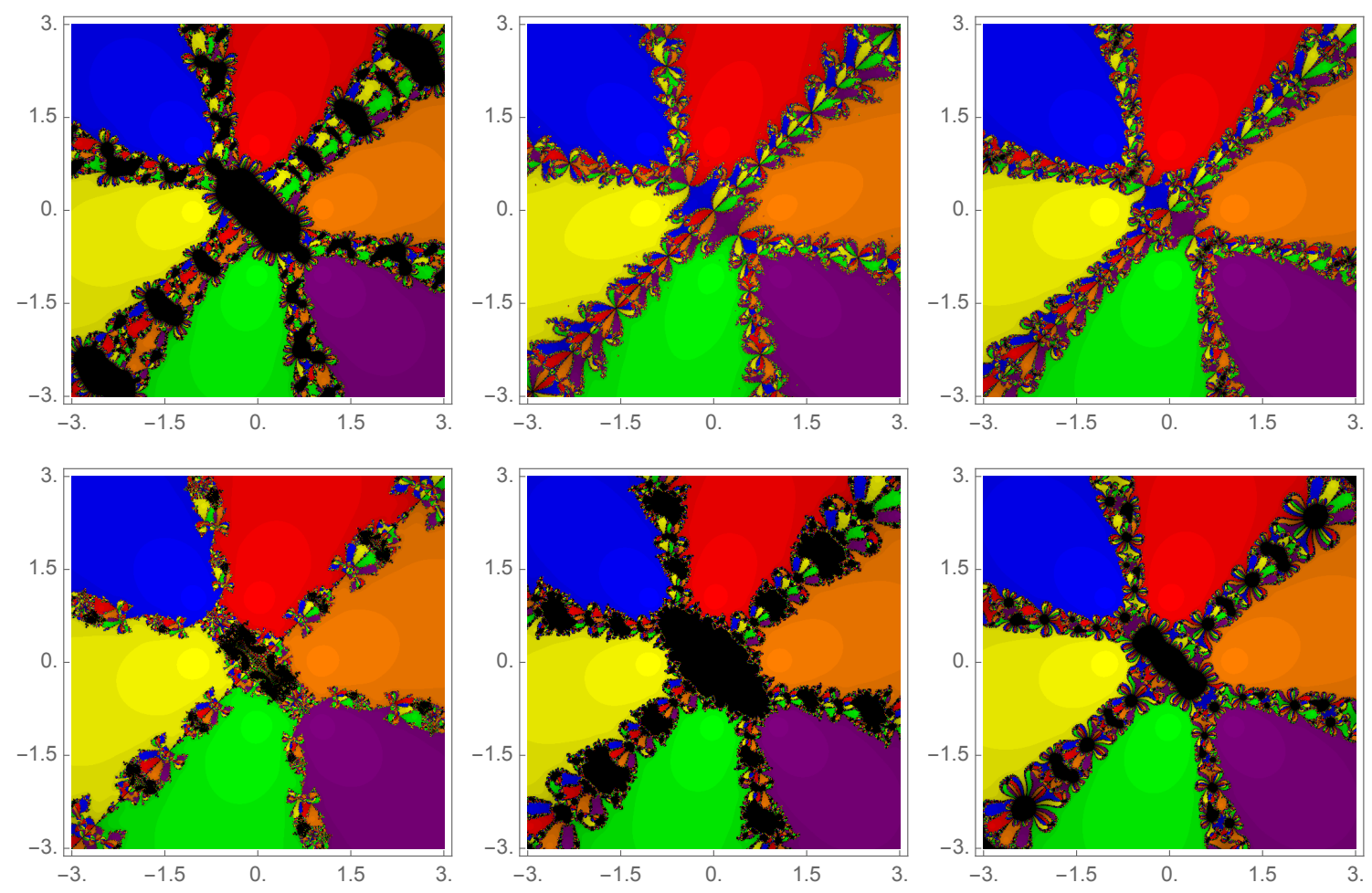

Figure 4: Comparison of basins of attraction of methods (2.1) and (2.7)-2.11) for the test problem $p_{4}(z)=\left(z^{4}-1\right)\left(z^{2}+2 i\right)=0$. 

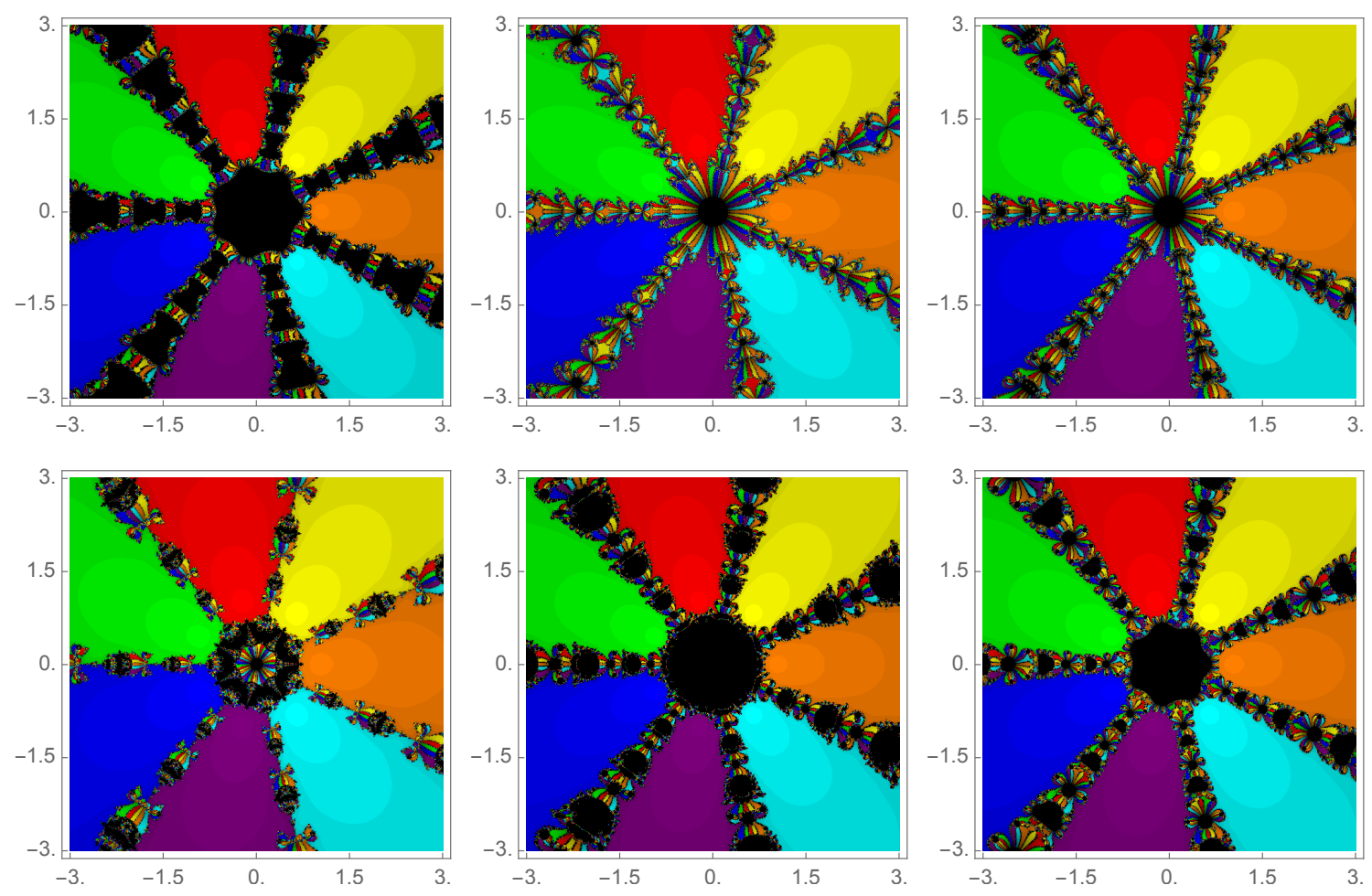

Figure 5: Comparison of basins of attraction of methods 2.1) and (2.7)-2.11) for the test problem $p_{5}(z)=z^{7}-1=0$.

starts in $z_{0}$. Hence, $z_{0}$ is not a good choice. Both the black zones and the zones with a lot of colors are not suitable for choosing the initial guess $z_{0}$ if precise root should be reached. Although the most attractive pictures appear when we have very intricate frontiers between basins of attraction, they correspond to the cases where the dynamic behavior of the method is more unpredictable and the method is more demanding with respect to choice of the initial point.

Finally, we have included in Table 4 the results of some numerical experiments to measure the behavior of the five iterative methods (2.1) and (2.7) -2.11) in finding the roots of the test polynomials $p_{j}, j=1, \ldots, 6$. To compute the data of this table, we have applied the six methods to the six polynomials, starting at an initial points $z_{0}$ on a $600 \times 600$ grid in the rectangle $[-3,3] \times[-3,3]$ of the complex plane. The same way was used in Figures 16 to show the basins of attraction of the roots. In particular, we decide again that an initial point $z_{0}$ has reached a root $z^{*}$ when its distance to $z^{*}$ is less than $10^{-3}$ (in this case $z_{0}$ is in the basin of attraction of $z^{*}$ ) and we decide that the method starting in $z_{0}$ diverges when no root is found in a maximum of 15 iterations of the method. We say in this case that $z_{0}$ is a "nonconvergent point". In Table 4, we have abbreviated the methods (2.1) and (2.7)-2.11) as M1-M6, respectively. The column I/P shows the mean of iterations per point until the algorithm decides that a root has been reached or the point is declared nonconvergent. The column NC shows the percentage of nonconvergent points, indicated as black zones in the pictures of Figures 1 6. It is clear that the nonconvergent points have a great influence on the values of I/P since these points contribute always with the maximum number of 15 allowed iterations. In contrast, "convergent points" are reached usually very fast due to the fact that we are dealing with methods of order eight. To reduce the effect of nonconvergent points, we have included the column $\mathrm{I}_{\mathrm{C}} / \mathrm{C}$ which shows the mean number of iterations per convergent point. If we use either the columns I/P or the column $\mathrm{I}_{\mathrm{C}} / \mathrm{C}$ to compare the performance of the iterative methods, we clearly obtain different conclusions. 

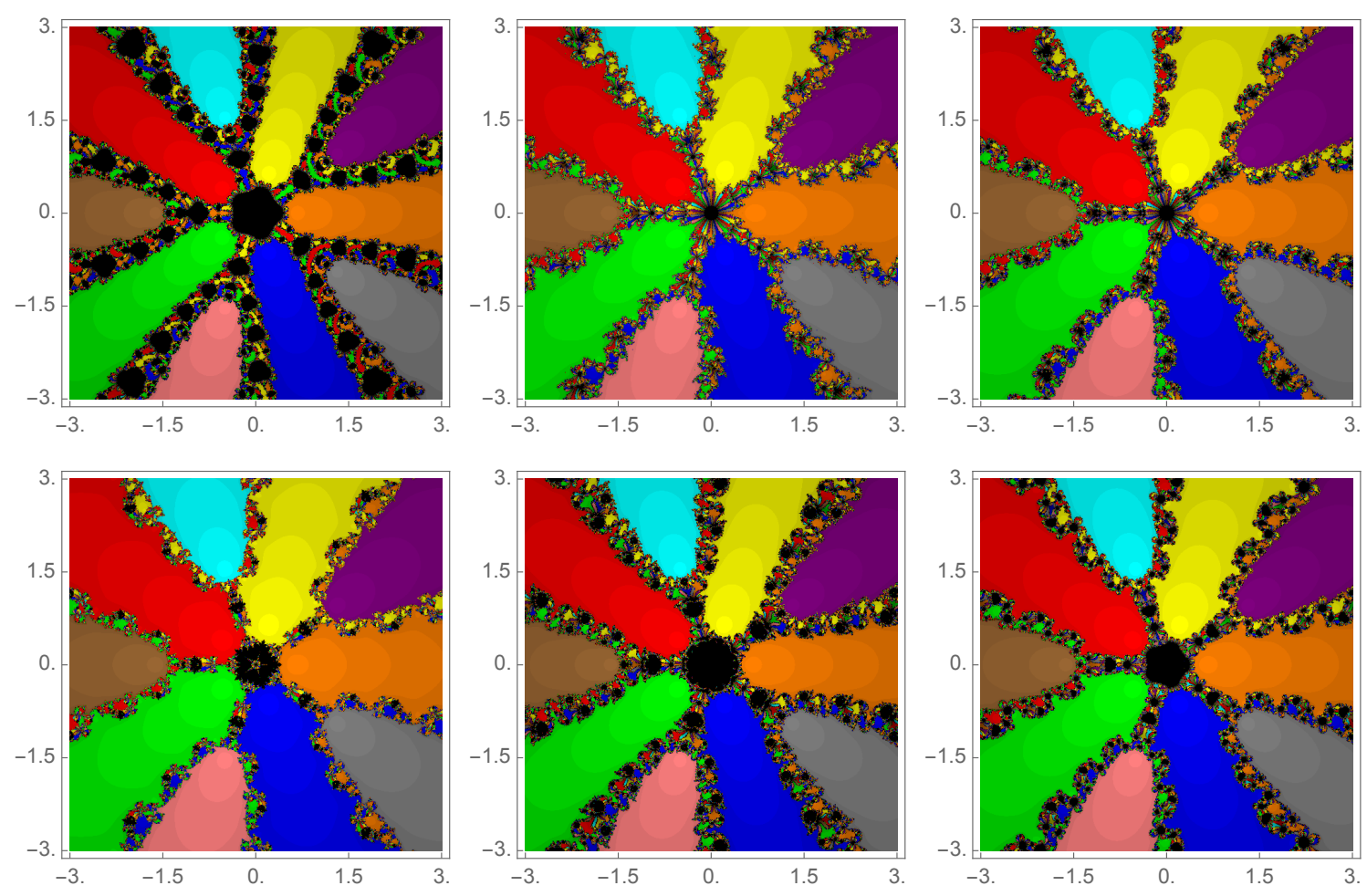

Figure 6: Comparison of basins of attraction of methods (2.1) and (2.7)-(2.11) for the test problem $p_{6}(z)=\left(10 z^{5}-1\right)\left(z^{5}+10\right)=0$.

\begin{tabular}{cclll}
\hline Polynomial & Method & $\mathrm{I} / \mathrm{P}$ & $\mathrm{NC}(\%)$ & $\mathrm{I}_{\mathrm{C}} / \mathrm{C}$ \\
\hline$p_{1}(z)$ & M1 & 2.21 & 0.00111 & 2.21 \\
& M2 & 2.19 & 0. & 2.19 \\
& M3 & 2.16 & 0. & 2.16 \\
& M4 & 2.11 & 0. & 2.11 \\
& M5 & 6.01 & 71.0 & 2.09 \\
& M6 & 2.30 & 0.0256 & 2.30 \\
\hline \multirow{2}{*}{$p_{2}(z)$} & M1 & 2.90 & 0.125 & 2.89 \\
& M2 & 2.88 & 0.00111 & 2.88 \\
& M3 & 2.82 & 0.00444 & 2.82 \\
& M4 & 2.73 & 0. & 2.73 \\
& M5 & 4.32 & 27.5 & 2.81 \\
& M6 & 3.21 & 0.216 & 3.18 \\
\hline \multirow{2}{*}{$p_{3}(z)$} & M1 & 3.22 & 0.802 & 3.13 \\
& M2 & 2.99 & 0.0178 & 2.99 \\
& M3 & 2.94 & 0.0367 & 2.94 \\
& M4 & 2.82 & 0. & 2.82 \\
& M5 & 3.28 & 5.47 & 2.99 \\
& M6 & 3.42 & 1.08 & 3.30 \\
\hline
\end{tabular}

\begin{tabular}{cclll}
\hline Polynomial & Method & $\mathrm{I} / \mathrm{P}$ & $\mathrm{NC}(\%)$ & $\mathrm{I}_{\mathrm{C}} / \mathrm{C}$ \\
\hline$p_{4}(z)$ & M1 & 6.00 & 17.7 & 4.06 \\
& M2 & 4.06 & 0.819 & 3.97 \\
& M3 & 4.21 & 1.82 & 4.01 \\
& M4 & 3.95 & 4.40 & 3.44 \\
& M5 & 4.44 & 20.0 & 3.57 \\
& M6 & 5.17 & 9.35 & 4.15 \\
\hline$p_{5}(z)$ & M1 & 6.89 & 24.4 & 4.27 \\
& M2 & 4.81 & 3.33 & 4.46 \\
& M3 & 5.07 & 5.70 & 4.46 \\
& M4 & 4.59 & 7.05 & 3.80 \\
& M5 & 5.02 & 21.4 & 4.02 \\
& M6 & 5.78 & 13.3 & 4.36 \\
\hline$p_{6}(z)$ & M1 & 6.72 & 18.2 & 4.88 \\
& M2 & 4.68 & 2.29 & 4.44 \\
& M3 & 4.89 & 4.04 & 4.46 \\
& M4 & 4.44 & 3.96 & 4.00 \\
& M5 & 5.26 & 11.8 & 4.71 \\
& M6 & 5.45 & 8.49 & 4.56 \\
\hline
\end{tabular}

Table 4: Measures of convergence of the iterative methods (2.1) and (2.7)-(2.11) (abbreviated as M1-M6) applied to find the roots of the polynomials $p_{j}(z), j=1, \ldots, 6$. 


\section{Conclusion}

We have introduced a new optimal three-point method without memory for approximating a simple root of a given nonlinear equation which use only four function evaluations each iteration and result in a method of convergence order eight. Therefore, Kung and Traub's conjecture is supported. Numerical examples and comparisons with some existing eighth-order methods are included and confirm the theoretical results. The numerical experience suggests that the new method is a valuable alternative for solving these problems and finding simple roots. We used the basins of attraction for comparing the iterative algorithms and we have included some tables with comparative results.

Acknowledgments. The research of the fourth author is supported by grant MTM2015-65888C4-4 from DGI (Spanish Government).

\section{References}

[1] Amat, S., Busquier, S., Magreñán, Á.A.: Reducing chaos and bifurcations in Newton-type methods, Abstr. Appl. Anal. 2013, Art. ID 726701, 10 pages (2013).

[2] Babajee, D.K.R., Cordero, A., Soleymani, F., Torregrosa, J.R.: On improved three-step schemes with high efficiency index and their dynamics, Numer. Algorithms 65, 153-169 (2014).

[3] Bi, W., Ren, H., Wu, Q.: Three-step iterative methods with eighth-order convergence for solving nonlinear equations, J. Comput. Appl. Math. 225, 105-112 (2009).

[4] Chicharro, F., Cordero, A., Gutiérrez, J.M., Torregrosa, J.R.: Complex dynamics of derivative-free methods for nonlinear equations, Appl. Math. Comput. 219, 7023-7035 (2013).

[5] Chun, C., Lee, M.Y.: A new optimal eighth-order family of iterative methods for the solution of nonlinear equations, Appl. Math. Comput. 223, 506-519 (2013).

[6] Cordero, A., Torregrosa, J.R.: Variants of Newton's method using fifth-order quadrature formulas, Appl. Math. Comput. 190, 686-698 (2007).

[7] Ezquerro, J.A., Hernández, M.A.: An improvement of the region of accessibility of Chebyshev's method from Newton's method, Math. Comp. 78, 1613-1627 (2009).

[8] Ezquerro, J.A., Hernández, M.A.: An optimization of Chebyshev's method, J. Complexity 25, 343-361 (2009).

[9] Ferrara, M., Sharifi, S., Salimi, M.: Computing multiple zeros by using a parameter in Newton-Secant method, SeMA Journal, accepted (2016).

[10] Grau-Sánchez, M., Noguera, M., Gutiérrez, J.M.: On some computational orders of convergence, Appl. Math. Lett. 23(4), 472-478 (2010).

[11] Gutiérrez, J.M., Magreñán, Á.A., Varona, J.L.: The "Gauss-Seidelization" of iterative methods for solving nonlinear equations in the complex plane, Appl. Math. Comput. 218, 2467-2479 (2011).

[12] Hernández-Paricio, L.J., Marañón-Grandes, M., Rivas-Rodríguez, M.T.: Plotting basins of end points of rational maps with Sage, Tbil. Math. J. 5(2), 71-99 (2012).

[13] Jarratt, P.: Some fourth order multipoint iterative methods for solving equations, Math. Comp. 20, 434-437 (1966).

[14] King, R.F.: A family of fourth order methods for nonlinear equations, SIAM J. Numer. Anal. 10, 876-879 (1973). 
[15] Kung, H.T., Traub, J.F.: Optimal order of one-point and multipoint iteration, J. Assoc. Comput. Mach. 21, 634-651 (1974).

[16] Lotfi, T., Sharifi, S., Salimi, M., Siegmund, S.: A new class of three-point methods with optimal convergence order eight and its dynamics, Numer. Algorithms 68, 261-288 (2015).

[17] Neta, B.: On a family of multipoint methods for nonlinear equations, Internat. J. Comput. Math. 9, 353-361 (1981).

[18] Neta, B., Chun, C., Scott, M.: Basins of attraction for optimal eighth order methods to find simple roots of nonlinear equations, Appl. Math. Comput. 227, 567-592 (2014).

[19] Ostrowski, A.M.: Solution of Equations and Systems of Equations, 2nd ed., Academic Press, New York (1966).

[20] Petković, M.S., Neta, B., Petković, L.D., Džunić, J.: Multipoint Methods for Solving Nonlinear Equations, Elsevier/Academic Press, Amsterdam (2013).

[21] Sharifi, S., Ferrara, M., Salimi, M., Siegmund, S.: New modification of Maheshwari method with optimal eighth order of convergence for solving nonlinear equations, preprint (2015).

[22] Sharifi, S., Siegmund, S., Salimi, M.: Solving nonlinear equations by a derivative-free form of the King's family with memory, Calcolo, doi: 10.1007/s10092-015-0144-1 (2015).

[23] Sharifi, S., Salimi, M., Siegmund, S., Lotfi, T.: A new class of optimal four-point methods with convergence order 16 for solving nonlinear equations, Math. Comput. Simulation 119, 69-90 (2016).

[24] Sharma, J.R., Sharma, R.: A new family of modified Ostrowski's methods with accelerated eighth order convergence, Numer. Algorithms 54, 445-458 (2010).

[25] Stewart, B.D.: Attractor Basins of Various Root-Finding Methods, M.S. thesis, Naval Postgraduate School, Monterey, CA (2001).

[26] Thukral, R., Petković, M.S.: A family of three-point methods of optimal order for solving nonlinear equations, J. Comput. Appl. Math. 233, 2278-2284 (2010).

[27] Traub, J.F.: Iterative Methods for the Solution of Equations, Prentice Hall, Englewood Cliffs, N.J. (1964).

[28] Varona, J.L.: Graphic and numerical comparison between iterative methods, Math. Intelligencer 24(1), $37-46(2002)$.

[29] Wang, X., Liu, L.: New eighth-order iterative methods for solving nonlinear equations, J. Comput. Appl. Math. 234, 1611-1620 (2010).

[30] Weerakoon, S., Fernando, T.G.I.: A variant of Newton's method with accelerated third-order convergence, Appl. Math. Lett. 13(8), 87-93 (2000). 